\title{
Fourth Pharyngeal Pouch
}

National Cancer Institute

\section{Source}

National Cancer Institute. Fourth Pharyngeal Pouch. NCI Thesaurus. Code C34181.

A paired evag ination of embryonic pharyngeal endoderm, which form the superior parathyroid glands. 